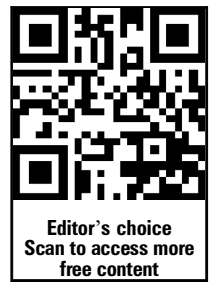

- Additional material is published online only. To view please visit the journal online (http://dx.doi.org/10.1136/ heartjnl-2013-304479).

${ }^{1}$ Quality and Outcomes Research Unit, University Hospital Birmingham, Birmingham, UK

${ }^{2}$ School of Clinical and Experimental Medicine, University of Birmingham, Birmingham, UK

${ }^{3}$ Department of Primary Care \& Population Health, University College London, London, UK

\section{Correspondence to} Professor Domenico Pagano, Department of Cardiothoracic Surgery, University Hospital Birmingham, Queen Elizabeth Hospital, Edgbaston, Birmingham B15 2TH, UK: domenicopagano@me.com

Received 21 June 2013 Revised 21 August 2013

Accepted 22 August 2013 Published Online First 16 September 2013

\title{
Fatal venous thromboembolism associated with hospital admission: a cohort study to assess the impact of a national risk assessment target
}

\author{
Will Lester, ${ }^{1,2}$ Nick Freemantle, ${ }^{1,3}$ Irena Begaj, ${ }_{1}^{1}$ Daniel Ray ${ }_{1}{ }^{1}$ John Wood ${ }^{3}$ \\ Domenico Pagano ${ }^{1,2}$
}

\begin{abstract}
Objectives In 2010, the Department of Health in England introduced an incentivised national target for National Health Service (NHS) hospitals aiming to increase the number of patients assessed for the risk of developing venous thromboembolism (VTE) associated with hospital admission. We assessed the impact of this initiative on VTE mortality and subsequent readmission with non-fatal VTE.
\end{abstract}

Design Observational cohort study.

Patients All patients admitted to NHS hospitals in

England between July 2010 and March 2012.

Interventions An NHS hospital which assessed at least $90 \%$ of patient admissions achieved the quality standard.

Main outcome measures The principal outcome measured was death from VTE up till 90 days after hospital discharge using linked Office of National Statistics and Hospital Episode Statistics data.

Results In the principal analyses of patients admitted to hospital for more than 3 days, there was a statistically significant reduction in VTE deaths in hospitals achieving 90\% VTE risk assessment: relative risk (RR) 0.85 (95\%

$\mathrm{Cl} 0.75$ to $0.96 ; p=0.011$ ) for VTE as the primary cause of death. In supportive analyses of postdischarge deaths after index admissions of up to 3 days, there was also a reduction in fatal VTE RR 0.61 (0.48 to 0.79; $p=0.0002$ ). This effect was seen for both surgical and non-surgical patients. No effect was seen in day case admissions. There was no change in non-fatal VTE readmissions up to 90 days after discharge.

Conclusions A national quality initiative to increase the number of hospitalised patients assessed for risk of VTE has resulted in a reduction in VTE mortality.

\section{INTRODUCTION}

Hospital associated venous thromboembolism (VTE) is an important and potentially preventable cause of death and disability. In the Million Women Study cohort, ${ }^{1}$ the relative risk (RR) of VTE increased more than a 100-fold after hospital admissions for surgical procedures and persisted up to 90 days from the admission; thus, many deaths are expected to occur after discharge from hospital and any VTE event within 90 days may be associated with hospital admission. Deaths from VTE are often sudden or are misdiagnosed premortem and prevention is a key strategy. ${ }^{2}$ Appropriate use of thromboprophylaxis was described as the 'number one patient safety practice' for US hospitals. $^{3}$ In 2010, the Department of Health in England introduced a national quality initiative (commissioning for quality innovation; CQUIN) ${ }^{4}$ which imposed financial penalties for hospitals failing to perform VTE risk assessment in at least $90 \%$ of their patients using a defined risk assessment tool. ${ }^{5}$ In conjunction with national guidelines and quality standards produced by the National Institute for Health and Care Excellence, it was expected that patients identified with risk factors for VTE would receive appropriate interventions to reduce their risk (including patient information and mechanical and/or pharmacological thromboprophylaxis). However, this national policy has generated some controversy, particularly in relation to medical patients. ${ }^{67}$

The true magnitude of the problem associated with hospital acquired VTE is not well described empirically, and the effectiveness of strategies to reduce its risks are unknown outside of the context of clinical trials. The aim of this study was to assess the impact of the incentivised policy of risk assessment for VTE on hospital-associated VTE mortality and VTE readmission.

\section{METHODS}

Analysis was based upon a series of monthly observations from each National Health Service (NHS) hospital trust in England, and the association between outcomes and the achievement or otherwise of the VTE risk assessment target in that month. Patients were included in the analysis based on their date of admission. In the principal analyses, we included all patients admitted to hospital for more than 3 days, excluding all deaths occurring before the fourth day from admission and any fatality occurring when Hospital Episode Statistics (HES) coding for the admission contained an International Classification of Diseases (ICD10) VTE code in position one (as this would represent delayed death in a patient admitted with VTE rather than death from VTE occurring during or after the index hospital admission). VTE mortality was described as 'inhospital' or 'postdischarge' (within 90 days after discharge) and as a total of these two periods (ie, deaths which occurred more than 3 days after admission and within 90 days of discharge).

Summary hospital level data on risk assessment were obtained from monthly submissions to the UNIFY2 database, the database used by the Department of Health in England to monitor 
performance and trigger incentive payments. Data were extracted on 9 November 2012 by two analysts for quality control. The population studied was all patients admitted to an NHS hospital trust in England $(n=163)$ between July 2010 and March 2012, accessed through HES. The Office of National Statistics data, linked to HES, were used to identify mortality outcomes. Mortality outcomes were considered relevant if an ICD10 VTE code was listed in the first position of the death certificate ('primary VTE death'), thus where VTE was considered the direct cause of death, or anywhere within the first three positions ('VTE related death') where VTE is considered either the direct cause or a contributing cause of death. The ICD10 codes used are those specified by the NHS-Outcome Framework 2013/14: ${ }^{8}$ I260, I269, I800, I801, I802, I803, I808, I809, I821, I822, I823, I828, I829, O082, O223, O229, O870, $\mathrm{O} 871, \mathrm{O} 879$ and $\mathrm{O} 882$.

For events occurring after discharge, we excluded all patients with a HES ICD10 VTE code at any position in the coding of the index admission, thus including only new VTE events. More detailed information on criteria for patient selection is given in online supplementary table S1.

Supportive analyses on the impact of achieving the 90\% VTE risk assessment quality standard were performed on patients admitted with less than 4 days of hospital stay, on day case admissions, and by whether or not surgical procedures were undertaken during the index admission (according to ICD10 codes in HES). We also assessed the impact of the intervention on non-fatal hospital readmissions with an ICD10 VTE code at position one or two, thus where VTE was the primary or major contributing cause of the readmission. Finally, we assessed the extent to which our results were sensitive to the $90 \%$ risk assessment threshold imposed by the Department of Health (DH) by analysing the relationship between the $\log _{e}$ proportion of patients' risk assessed by trust each month against VTE outcomes. Two informatics experts extracted all data independently, and the resulting data sets were compared to ensure consistency.

\section{Statistical methods}

We fitted a Poisson mixed effects model to the counts of the events of interest (deaths, readmissions) for patients admitted in each of the 21 months of data for the 163 English NHS hospital trusts, using a log link function and with the $\log _{\mathrm{e}}$ of the number of admissions during that month as an offset (weighting) variable. We included a classification variable indicating whether or not the hospitals had achieved the benchmark quality standard of at least $90 \%$ of admitted patients being assessed for risk of VTE in that month. Because of the structure of the data, some of the information concerning the effect of achieving the quality standard is contained in comparisons between hospitals but this is also potentially confounded by organisational characteristics not directly related to the quality improvement programme, and some is contained in comparisons between months within hospital organisations. To allow for any consequent bias, hospitals were included in the model as random intercept terms. Since hospital organisations tended to maintain the quality standard once they had initially attained it, there is a partial confounding between that and any other (unknown) monotonic time trends, which might vary somewhat between hospitals. To allow efficiently for this in the model, an overall linear fixed effects variable for time was included along with an approximate low rank smoother for time as a random effect within hospitals. ${ }^{9}$ All analyses were conducted in SAS V.9.2 (SAS Institute, Cary, North Carolina, USA).

\section{RESULTS}

Across the 21-month period of the study, on average, hospitals achieved the quality standard $(\geq 90 \%$ of admitted patients assessed for VTE risk) $56 \%$ of the time. There was a substantial improvement over the time period in the raw VTE screening score supplied by hospital organisations to the Unify 2 database. In July 2010 (month 1), the median hospital rate of assessment for VTE risk was $51 \%$, the 25 percentile trust achieved $27 \%$ and the 75 percentile trust achieved $71 \%$ patients assessed. In March 2012 (the 21st month), the median hospital rate of assessment for VTE risk was $93 \%$, the 25 percentile was $91 \%$ and the 75 percentile was $96 \%$. However, there was also significant variation between hospitals. Over the 21-month period, the median proportion of time for which the quality target was achieved was $13 / 21$ months (62\%), with lower and upper quartiles of $8 / 21$ months $(38 \%)$ and $15 / 21$ months (71\%), respectively. Further, out of the 163 included hospital trusts, there were nine which never achieved the quality targets, and nine which always achieved them.

\section{Principal analyses: effect of achieving VTE risk assessment target in admissions with $>3$ days hospital stay}

In our principal analyses of patients with more than 3 days inhospital stay, we included data from 4141041 admissions, which were associated with 8578 VTE readmissions. Inhospital VTE related mortality occurred in 4334 patients, and primary VTE mortality occurred in 1318 patients. There were 1651 VTE related deaths within 90 days of discharge, of which 895 were primary VTE deaths. Figure 1 shows the distribution of primary VTE deaths over time and whether deaths occurred during admission (red line) or after discharge (blue line).

In the principal analyses, achieving VTE risk assessment in $\geq 90 \%$ of patients admitted to hospital was associated with a reduction in mortality within 90 days of discharge for primary VTE deaths (RR $0.81 ; 95 \%$ CI 0.67 to $0.97 ; p=0.026$ ), for total primary VTE deaths both inhospital and within 90 days from discharge (RR $0.85 ; 0.75$ to $0.96 ; p=0.011$ ) and for total deaths where VTE was in any of the first three positions on the death certificate (RR $0.92 ; 95 \%$ CI 0.85 to $0.99 ; p=0.033$ ) (figure 2). In contrast, achieving the quality standard had no effect on the rate of non-fatal VTE readmission within 90 days (RR $1.04 ; 95 \%$ CI 0.97 to $1.11 ; p=0.301$ ). Inhospital mortality for primary VTE deaths (RR 0.86 ; 95\% CI 0.74 to 1.01 ; $\mathrm{p}=0.061$ ) or VTE related deaths (RR $0.92 ; 95 \%$ CI 0.84 to $1.00 ; \mathrm{p}=0.057)$ and VTE related deaths within 90 days of discharge (RR $0.91 ; 95 \%$ CI 0.79 to $1.05 ; \mathrm{p}=0.196$ ) did not reach statistical significance in isolation.

\section{Supportive analyses}

Patients with less than 4 days hospital stay and day cases

We examined the effect of achieving 90\% VTE risk assessment on primary and VTE related deaths within 90 days of discharge in patients with hospital length of stay of less than 4 days and in day cases. There were 13571420 admissions with less than 4 days stay excluding day cases. In this cohort, there were 874 VTE related deaths, and 512 primary VTE deaths. There were 9534178 day cases with 393 VTE related deaths and 192 primary VTE deaths.

Achieving the VTE risk assessment target was significantly associated with a reduction in death within 90 days of discharge both for primary VTE deaths (RR $0.61 ; 95 \%$ CI 0.48 to 0.79 ; $\mathrm{p}=0.0002$ ) and for VTE related deaths (RR 0.74; 95\% CI 0.61 to $0.90 ; p=0.003$ ) in patients with less than 4 days hospital stay 


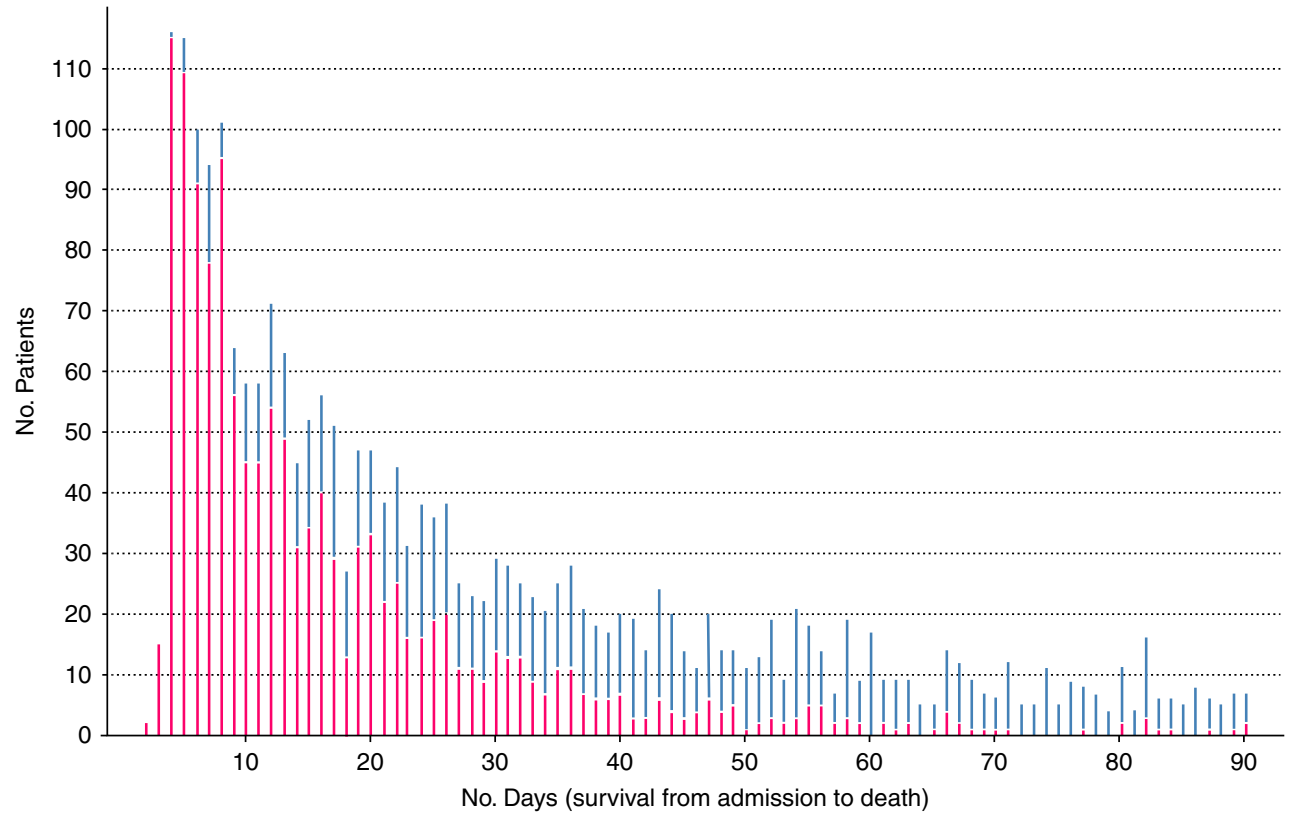

Figure 1 Histogram to indicate time to fatality with venous thromboembolism code position one of the death certificate after 3 days of hospital admission until 90 days after discharge. The blue bars represent deaths after discharge. Access the article online to view this figure in colour.

(figure 3). There was no evidence of an effect of achieving the VTE assessment target on deaths within 90 days among day cases however (figure 4).

Effect of implementing VTE risk assessment policy on surgical and non-surgical admissions

We examined the effect of implementing the VTE risk assessment policy on patients by whether surgical procedures were undertaken (surgical group) or not (non-surgical group) during the index admission. We analysed data on patients with a hospital stay of more than 3 days, and those less than 4 days (excluding day cases) (see table 1). Numerically, VTE events in the surgical group were lower in comparison with the nonsurgical group. We also analysed the data for day cases shown in figure 4 separately for the surgical and non-surgical groups and found no evidence of an effect in either (data not shown).

\section{Non-surgical group}

For 2590547 non-surgical admissions with more than 3 days hospital stay, implementing the VTE risk assessment policy did not influence VTE related deaths within 90 days of discharge. There were 1135 such events and the CIs on the estimates were reasonably narrow. Primary VTE deaths within 90 days of discharge and VTE related inhospital deaths were all associated with around $10 \%$ reduction in risk, and the reduction in VTE related inhospital deaths was modestly statistically significant.

Among 10719502 non-surgical admissions with less than 4 days hospital stay (excluding day cases), we saw strong and

Relative Risk (95\% Cl; p: $n=$ events)

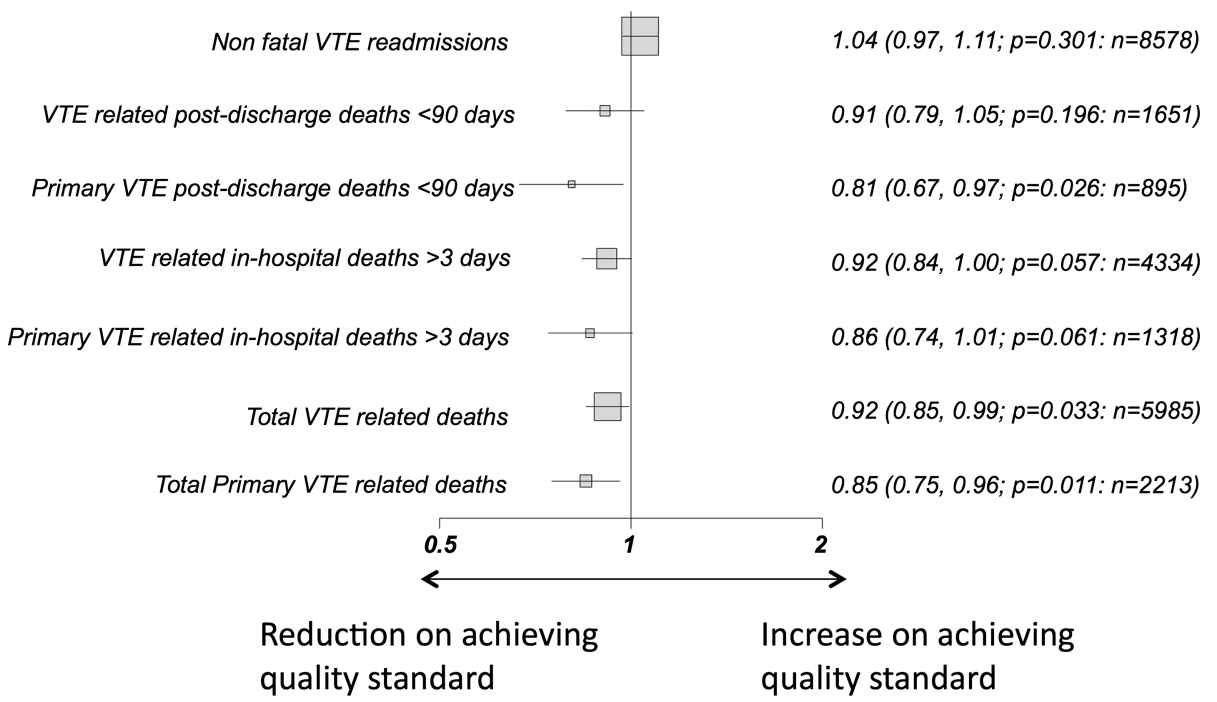

Figure 2 Principal analysis examining the impact of achieving the venous thromboembolism (VTE) risk assessment quality standard on mortality outcomes and non-fatal VTE readmissions for patients admitted to hospital $>3$ days. Relative risks and $95 \%$ Cls. 


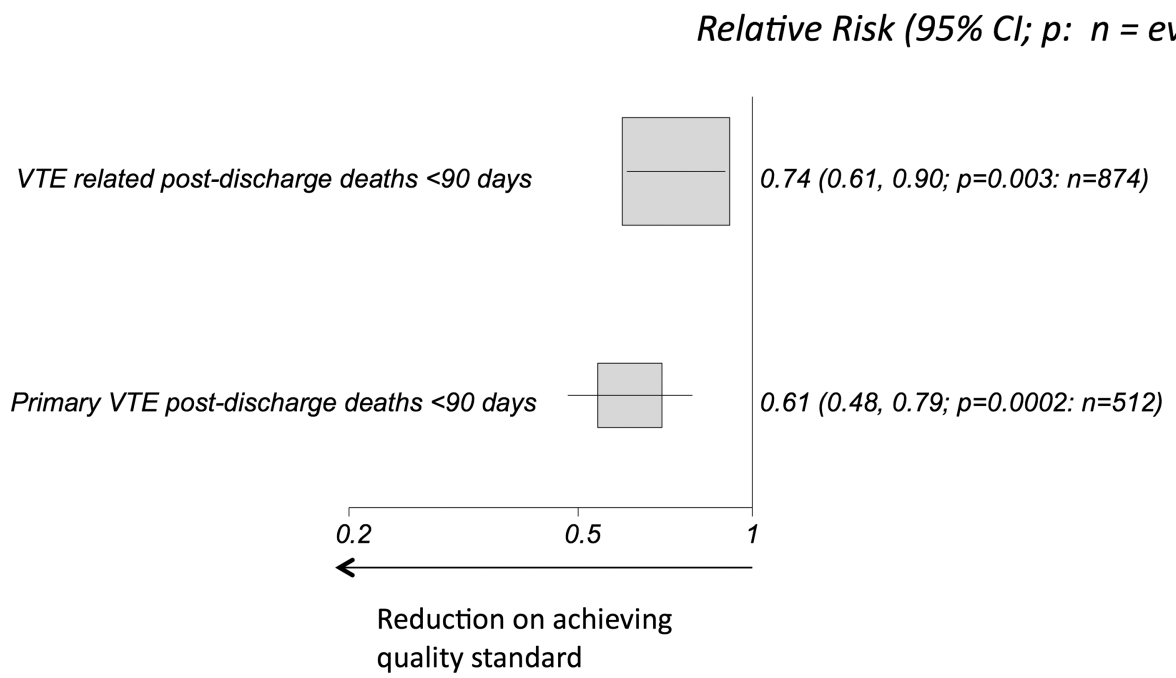

Figure 3 Effect of achieving venous thromboembolism (VTE) risk assessment quality standard on mortality outcomes within 90 days of discharge in admissions $<4$ days. Relative risks and $95 \%$ Cls.

convincing reductions in deaths within 90 days of discharge for both primary VTE and VTE related deaths.

\section{Surgical group}

In 1550794 admissions coded for a surgical room procedure with greater than 3 days hospital stay, we found no evidence of a convincing effect of implementing the VTE risk assessment policy on inhospital VTE mortalities (primary or related), but primary VTE deaths within 90 days of discharge were significantly reduced in this group. The risk of VTE related death within 90 days of discharge was reduced by $18 \%$, but was not statistically significant although the number of contributing events was modest.

In 2851838 surgical admissions less than 4 days (excluding day cases), the number of deaths for all analyses was modest leading to quite wide CIs. All the RR values were less than one.

Examining the proportion of patients risk assessed

In examining the proportion of risk assessments performed in each hospital trust by month rather than by the $\geq 90 \%$ target, all analysis provided qualitatively similar results to those seen in figure 2. For example, the total effect on primary VTE death in the principle analysis provided an estimate RR 0.88 (95\% CI 0.79 to $0.98 ; \mathrm{p}=0.02$ ).

\section{DISCUSSION}

In 2010, the Department of Health in England introduced a quality incentive for NHS hospital trusts to screen at least $90 \%$ of admitted patients for the risk of developing VTE. We found that the achievement of this quality standard was associated with a significant overall reduction in mortality due to VTE (inhospital and within 90 days from discharge). This finding was detected in patients with hospital stay greater than 3 days, whom we judged most likely to be at risk of developing VTE and most likely to receive thromboprophylaxis, and for admissions with hospital stay less than 4 days. We did not detect any effect on day case VTE mortality. Numerically, VTE events were more common in patients not undergoing a surgical room procedure during their index admission, although there are considerably more of these admissions and the overall risk is similar.

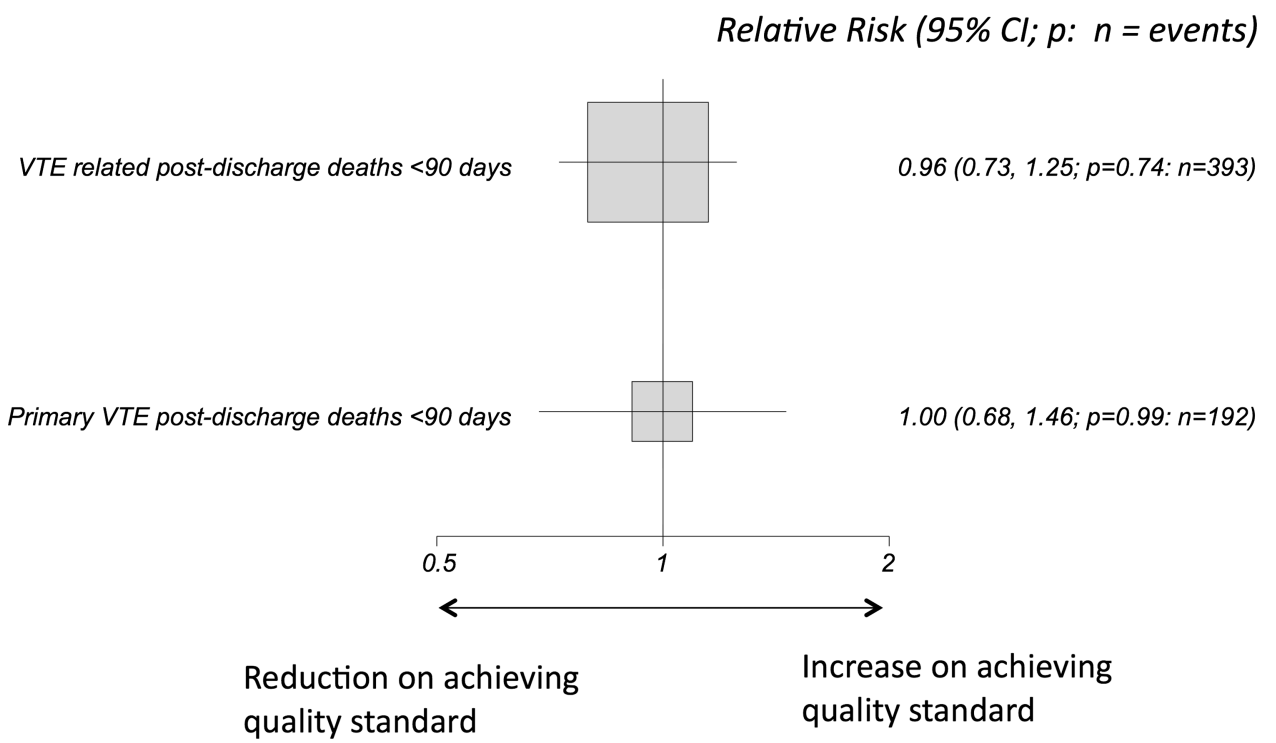

Figure 4 Effect of achieving venous thromboembolism (VTE) risk assessment quality standard on mortality outcomes within 90 days of discharge in day cases. Relative risks and $95 \%$ Cls. 
Table 1 Effect of achieving venous thromboembolism (VTE) risk assessment quality target on patients by whether they were coded for a surgical room procedure or not during the index admission, examining those patients admitted for more than 3 days, and those admitted for less than 4 days (excluding day cases)

\begin{tabular}{|c|c|c|c|c|c|}
\hline & \multirow[b]{2}{*}{ Relative risk } & \multicolumn{2}{|c|}{$95 \% \mathrm{Cl}$} & \multirow[b]{2}{*}{$\mathrm{p}$ Value } & \multirow[b]{2}{*}{$\mathrm{N}$ events } \\
\hline & & Lower & Upper & & \\
\hline \multicolumn{6}{|c|}{ Non-surgical admissions >3 days $n=2590547$} \\
\hline VTE deaths postdischarge & 0.963 & 0.814 & 1.138 & 0.653 & 1135 \\
\hline Primary VTE deaths postdischarge & 0.886 & 0.714 & 1.099 & 0.269 & 669 \\
\hline VTE inhospital deaths & 0.900 & 0.813 & 0.997 & 0.044 & 3302 \\
\hline Primary VTE inhospital deaths & 0.858 & 0.724 & 1.018 & 0.079 & 1065 \\
\hline All VTE deaths & 0.916 & 0.838 & 1.002 & 0.056 & 4437 \\
\hline All primary VTE deaths & 0.872 & 0.760 & 1.002 & 0.053 & 1734 \\
\hline \multicolumn{6}{|c|}{ Non-surgical admissions $<4$ days $n=10719502$} \\
\hline VTE deaths postdischarge & 0.743 & 0.602 & 0.918 & 0.006 & 761 \\
\hline Primary VTE deaths postdischarge & 0.617 & 0.472 & 0.808 & 0.001 & 450 \\
\hline \multicolumn{6}{|c|}{ Surgical admissions $>3$ days $n=1550794$} \\
\hline VTE deaths postdischarge & 0.816 & 0.646 & 1.031 & 0.088 & 516 \\
\hline Primary VTE deaths postdischarge & 0.624 & 0.440 & 0.884 & 0.008 & 226 \\
\hline VTE inhospital deaths & 0.970 & 0.819 & 1.149 & 0.723 & 1032 \\
\hline Primary VTE inhospital deaths & 0.919 & 0.670 & 1.259 & 0.596 & 253 \\
\hline All VTE deaths & 0.922 & 0.799 & 1.063 & 0.260 & 1548 \\
\hline All primary VTE deaths & 0.778 & 0.611 & 0.992 & 0.043 & 479 \\
\hline \multicolumn{6}{|c|}{ Surgical admissions $<4$ days $n=2851838$} \\
\hline VTE deaths postdischarge & 0.730 & 0.459 & 1.162 & 0.184 & 113 \\
\hline Primary VTE deaths postdischarge & 0.568 & 0.303 & 1.067 & 0.078 & 62 \\
\hline
\end{tabular}

A strength of our analyses is that we accounted efficiently for time effects using random effects radial smoothers. We also included trusts as random intercept terms. This is important as trusts were not the same in patient characteristics (including eye hospitals and tertiary specialist centres for other conditions along with district general hospitals), which was highlighted by the fact that nine hospital organisations never achieved the quality standard for VTE risk screening during the study period, and nine trusts achieved the standard for the entire period.

One potential bias is that a reduction in deaths in those who have survived to day 4 of an admission from VTE requires them to have survived for the first 3 days, and early deaths could be a form of competing risks, that is, an increase in early deaths (before 4 days) could give the impression of a decrease in later deaths (from day 4). We assessed this by examining VTE inhospital mortality (including all deaths which occurred in hospital) for subjects with admissions less than 4 days. These analyses showed a non-significant reduction in all VTE deaths (RR 0.95; $95 \%$ CI 0.84 to $1.08 ; \mathrm{p}=0.46$ ) and in deaths with VTE in the primary position on the death certificate (RR 0.89 ; 95\% CI 0.75 to $1.07 ; p=0.21$, thus providing no evidence of competing risks. A further potential limitation is the accuracy of coding which is controversial. ${ }^{10}$ This is more likely to be an issue with HES admission coding rather than death certification, which although flawed, ${ }^{11}$ represents a more concrete outcome determined by a clinician involved in patient care or else a coroner's postmortem determining the cause of death. Our methodology was designed to identify hospital associated VTE mortality occurring during an admission after more than 3 days with exclusion of patients where VTE was the primary reason for admission. As the effectiveness of this method relies on the quality of coding in hospitals across the country (which cannot be individually tested in our study), an unknown number of community acquired events with delayed death could have been assigned as hospital associated. There has been no step change in the hospital management of community acquired VTE over the study period and so this should not impact on the interpretation of our results. Other important considerations are that a VTE death within 90 days of discharge may not be directly attributable to the hospital admission itself but related to patient comorbidities and many events occur despite using thromboprophylaxis according to local protocols. ${ }^{12}$ Therefore, events which are truly preventable by VTE risk assessment and appropriate thromboprophylaxis are an unknown proportion of this cohort.

In our analysis plan we defined the achievement of the $90 \%$ VTE screening assessment, which helps avoid a financial penalty for the hospital organisation, as the main explanatory variable, as this was the standard identified by the programme. However, if instead we took the actual quality score by month as the explanatory variable in supportive analyses, we found a qualitatively similar pattern of results. Because we do not know which individual patients were risk-assessed and which were not in each hospital organisation, we cannot (without making considerable additional assumptions) estimate directly the effect on absolute VTE risk of conducting a risk-assessment on an individual patient or number needed to treat (NNT) to save a life.

Our data do not allow an indepth analysis of the mechanisms underlying the reduction in mortality. Making clinicians aware of patient VTE risk during hospital admission has been shown to reduce VTE events. ${ }^{13}{ }^{14}$ It is possible that introduction of the CQUIN target is associated with increasing use of pharmacological thromboprophylaxis and with increasing staff and patient awareness of the symptoms and signs of VTE and the adoption of simple non-pharmacological strategies to reduce the risk. This may explain the reduced mortality in the patients admitted for less than 4 days as it is likely that most of these patients receive little in the way of pharmacological prophylaxis. Increased awareness of VTE symptoms might also explain the 
apparent reduction in VTE mortality without an observed reduction in non-fatal VTE readmissions (which may be paradoxically increased). It has been estimated that about $60 \%$ of patients with fatal VTE have prodromal symptoms which are often misinterpreted, representing a missed opportunity to prevent a fatal outcome. ${ }^{2}$ In this study, the reduction of primary VTE deaths associated with $\geq 90 \%$ risk assessment in patients admitted for more than 3 days not having a surgical procedure seems to occur during admission but not clearly after discharge. Postdischarge pharmacological thromboprophylaxis is rarely given to this group of patients (unlike high risk surgical and obstetric patients) and transition to a reduced VTE risk may not be quite so apparent as with surgical patients after discharge. In contrast, it is postdischarge primary VTE deaths which appear to have been prevented in those having surgical procedures requiring admission for more than 3 days. Pharmacological thromboprophylaxis gives a greater degree of VTE risk reduction in surgical patients than in medical patients $(70 \%$ vs $50 \%)^{15} 16$ and these patient groups differ in terms of comorbidities, VTE risk factors and response to treatment. In trials of pharmacological prophylaxis in medical patients, no reduction of overall mortality has been detected. ${ }^{17}$ In our analysis of large numbers of 'real world' patients, a reduction in primary VTE mortality was detected. However, our study does not address the harms of any increase in pharmacological prophylaxis as a result of increased VTE risk assessment. Bleeding complications with pharmacological thromboprophylaxis may offset the benefits, particularly in medical patients. ${ }^{18}$

The aim of the VTE CQUIN in England was to reduce avoidable death, disability and chronic ill health from VTE. ${ }^{4}$ Achieving the target of $90 \%$ or more patients' risk assessment avoided a financial penalty of approximately $£ 0.5$ million a year for many acute hospital trusts. The CQUIN uses the risk of withholding money already allocated to a hospital baseline budget to drive specific quality targets. It is therefore a financially attractive method for commissioners of healthcare to achieve desired targets without financial investment, albeit at the risk of diverting attention away from healthcare outcomes not subject to such penalties. Our analyses provide convincing evidence of the effectiveness of the VTE risk screening programme in the English NHS. Taking the year 2011 as the basis for this estimation, if all trusts achieved the quality standard, we could expect as a result that 280 (95\% CI 25 to 532) deaths from VTE (anywhere in the first three positions on the death certificate) would have been avoided among patients with admissions greater than 3 days. In addition, we could expect that 150 deaths (95\% CI 58 to 225) within 90 days of discharge would have been prevented among subjects with admissions less than 4 days. It is likely that this is an underestimate of the true number of VTE fatalities ${ }^{19}$ and the low rate of postmortems preformed in England has been identified as contributing to this under-recognition. ${ }^{20}$

In summary, this study demonstrates that a national quality initiative to increase the number of patients screened for VTE risk to at least $90 \%$, linked to a financial penalty, has resulted in improvements in the outcome of death from VTE up to 90 days after hospital admission. Ideally, future similar initiatives should be delivered in conjunction with predefined methodology to assess their efficacy and impact. In terms of implications for clinical practice, this study reinforces the value of implementing strategies to increase the number of patients risk assessed for VTE on admission to hospital. Ideally, future research should attempt to assess the impact of increasing VTE risk assessment on patient and staff awareness and on the use of thromboprophylaxis and whether there are any measurable harms resulting from this.

Contributors WL: Conception, design, interpretation of data and drafting the manuscript. NF and JW: Conception, design, statistical analysis, interpretation of data and revision of content. IB: Conception, design, data extraction and revision of content. DR: Conception, data extraction and revision of content. DP: Conception, design and interpretation of data, revision of content, and final approval.

Funding QUORU is funded solely by University Hospital Birmingham NHS Foundation Trust.

Competing interests WL reports serving on advisory boards for Bayer and Boehringer Ingelheim and has received lecture fees from Bayer, Boehringer Ingelheim and Sanofi Aventis and received financial support for attendance of an educational meeting from Boehringer Ingelheim. NF has received funding for consulting, research and travel from Sanofi Aventis.

Ethics approval Institutional Study Registration was obtained by UHB-QE (CAB04920-12).

Provenance and peer review Not commissioned; externally peer reviewed.

\section{REFERENCES}

1 Sweetland S, Green J, Liu B, et al. Duration and magnitude of the postoperative risk of venous thromboembolism in middle aged women: prospective cohort study. BMJ 2009;339:b4583.

2 Cohen AT, Agnelli G, Anderson FA, et al. Venous thromboembolism (VTE) in Europe. The number of VTE events and associated morbidity and mortality. Thromb Haemost 2007:98:756-64.

3 Shojania KG, Duncan BW, McDonald KM, et al. Making health care safer: a critical analysis of patient safety practices. Evid Rep Technol Assess (Summ) 2001;43:1-668

4 http://www.dh.gov.uk/dr_consum_dh/groups/dh_digitalassets/documents/ digitalasset/dh_110431.pdf (accessed 15 Jun 2013)

5 http://www.dh.gov.uk/prod_consum_dh/groups/dh_digitalassets/@dh/@en/@ps/ documents/digitalasset/dh_113355.pdf (accessed 15 Jun 2013)

6 Welfare M. NICE's recommendations for thromboembolism are not evidence based. BMJ 2011;343:d6452.

7 D'Costa DF. Time to reconsider NICE guidance on heparin prophylaxis in medical inpatients. BMJ 2011;343:d7886.

8 https://www.gov.uk/government/uploads/system/uploads/attachment_data/file/ 213057/121109-Technical-Appendix.pdf (accessed 14 Aug 2013)

9 Ruppert D. Semiparametric regression. Cambridge; New York: Cambridge University Press, 2003.

10 Williams DR, Fuller JH, Stevens LK. Validity of routinely collected hospital admissions data on diabetes. Diabet Med 1989;6:320-4.

11 Nielson GP, Bjornsson J, Jonasson JG. The accuracy of death certificates. Virchows Archiv A Pathol Anat 1991;419:143-6.

12 Goldhaber SZ, Dunn K, MacDougall RC. New onset of venous thromboembolism among hospitalized patients at Brigham and Women's Hospital is caused more often by prophylaxis failure than by withholding treatment. Chest 2000;118:1680-4.

13 Piazza G, Rosenbaum EJ, Pendergast W, et al. Physician alerts to prevent symptomatic venous thromboembolism in hospitalized patients. Circulation 2009;119:2196-201.

14 Kucher N, Koo S, Quiroz R, et al. Electronic alerts to prevent venous thromboembolism among hospitalized patients. N Engl J Med 2005;352:969-77.

15 Collins R, Scrimgeour A, Yusuf $S$, et al. Reduction in fatal pulmonary embolism and venous thrombosis by perioperative administration of subcutaneous heparin. Overview of results of randomized trials in general, orthopedic, and urologic surgery. N Engl J Med 1988;318:1162-73.

16 Francis CW. Clinical practice. Prophylaxis for thromboembolism in hospitalized medical patients. N Engl J Med 2007;356:1438-44.

17 Kakkar AK, Cimminiello C, Goldhaber SZ, et al. Low-molecular-weight heparin and mortality in acutely ill medical patients. N Engl J Med 2011;365:2463-72.

18 Lederle FA, Zylla D, MacDonald R, et al. Venous thromboembolism prophylaxis in hospitalized medical patients and those with stroke: a background review for an American College of Physicians Clinical Practice Guideline. Ann Intern Med 2011:155:602-15.

19 Anderson FA Jr, Wheeler HB, Goldberg RJ, et al. A population-based perspective of the hospital incidence and case-fatality rates of deep vein thrombosis and pulmonary embolism. The Worcester DVT Study. Arch Intern Med 1991;151:933-8.

20 http://www.publications.parliament.uk/pa/cm200405/cmselect/cmhealth/99/99.pdf (accessed 15 Jun 2013) 